\title{
SARCOMATOID TUMOUR OF THE URINARY BLADDER- A CASE REPORT
}

\author{
Sumit Gupta1 ${ }^{1}$ Ashish Kumar'2, S. Rajendra Singh ${ }^{3}$
}

${ }^{1}$ Mch Student, Department of Urology, Regional Institute of Medical Sciences, Imphal. ${ }^{2}$ Mch Student, Department of Urology, Regional Institute of Medical Sciences, Imphal. ${ }^{3}$ Professor, Department of Urology, Regional Institute of Medical Sciences, Imphal.

HOW TO CITE THIS ARTICLE: Gupta S, Kumar A, Singh SR. Sarcomatoid tumour of the urinary bladder- a case report. J. Evolution Med. Dent. Sci. 2017;6(85):5928-5929, DOI: 10.14260/jemds/2017/1289

\section{PRESENTATION OF CASE}

Sarcomas are the most common mesenchymal tumour of the bladder, but are rare tumours and comprises only $1 \% 0^{1-2}$ of all bladder cancers. The average age of presentation is in the 6th decade of life and is twice more common in males. Importantly, bladder sarcomas are not smoking related like TCC of urinary bladder. Bladder sarcomas are usually highgrade, confined to bladder muscle. Treatment for localised disease includes radical cystectomy. ${ }^{3}$ Active chemotherapeutic regimens are lacking for bladder sarcomas. ${ }^{4}$

A 60-year-old female came to our centre with chief complaints of lower abdominal painful mass for three months, which was progressively increasing in size. She also complained of burning micturition and frequency of micturition. She was a non-smoker. Her past and family history was insignificant. On examination mass was firm, non-tender, smooth surface and fixed to underlying surface. Routine blood tests were within normal limits. On urine microscopy, there was pyuria and haematuria. CECT of whole abdomen showed well-defined enhancing lesion in the right inferolateral wall of bladder. Patient was prepared for total cystectomy, but patient refused, so partial cystectomy was done with $1 \mathrm{~cm}$ margin all around. Specimen was sent for histopathological examination. Macroscopically, mass was $20 * 15^{*} 11 \mathrm{~cm}$ and weight $1.73 \mathrm{~kg}$. HPE showed high-grade sarcoma, morphologically consistent with fibrosarcoma. On immunohistochemistry, tumour was desmin-negative and cytokeratin 8/18 negative. Patient was followed up to 6 months after which patient failed to come for followup.

\section{DIFFERENTIAL DIAGNOSIS}

Includes benign and malignant proliferation including pure sarcoma, particularly in cases composed exclusively of spindle cells, leiomyosarcoma, carcinoma with pseudosarcomatous stroma and sarcomas with pseudoepitheliomatous hyperplasia. ${ }^{5}$ In view of rarity of bladder sarcoma, any malignant spindle cell tumour in urinary bladder of an adult is considered sarcomatous carcinoma until proven otherwise. ${ }^{6}$

'Financial or Other Competing Interest': None.

Submission 22-08-2017, Peer Review 08-10-2017,

Acceptance 14-10-2017, Published 23-10-2017.

Corresponding Author:

Sumit Gupta,

A2/64, Ashiyana Colony,

Phase 2, Near RRK School,

Moradabad-244001,

Uttar Pradesh.

E-mail: sumitgupta3210@gmail.com

DOI: $10.14260 /$ jemds/2017/1289

\section{(c) (1) $(9)$}

\section{CLINICAL DIAGNOSIS}

The term sarcomatoid variant of urothelial carcinoma should be used for all biphasic malignant neoplasms exhibiting evidence of epithelial and mesenchymal differentiation with the presence or absence of heterologous elements. ${ }^{6}$ Various terms has been used for these neoplasms including carcinosarcoma. Sarcomatoid carcinoma is recommended terminology by WHO for such lesions and carcinosarcoma is used as synonym for these tumours.

Symptoms for these are same as of other urothelial tumours. Gross painless haematuria has been the most common symptom. Other common symptoms are dysuria, nocturia, acute urinary retention and lower abdominal pain. White elderly male are most commonly affected in their $7^{\text {th }}$ decade of life. No specific cause is known, but pelvic radiation and systemic chemotherapy especially cyclophosphamide for other malignancy is thought to be causative agents of bladder sarcomas.

Most common site of presentation is lateral walls of urinary bladder. It rarely arises from any other portion of urothelium. Cystoscopy usually shows broad based and often polypoid or papillary neoplasm rapidly growing into bladder lumen.

CT and MRI abdomen shows advanced stage of disease. ${ }^{6}$ Macroscopically, the tumour is typically large pedunculated. Oedema and necrosis are often evident surrounding the tumour. Gross extravesical soft tissue involvement is common. ${ }^{6}$ Patients usually present in advanced stage of disease. Most common site for metastatic disease is lung.

\section{PATHOLOGICAL DISCUSSION}

Histology reveals that tumour shows both malignant spindle cells and epithelial components. It may merge with foci of overlying CIS or with invasive urothelial carcinoma, small cell carcinoma or adenocarcinoma. Heterogeneous differentiation in the form of rhabdomyosarcomatous, chondrosarcomatous and osteosarcomatous may be present. Osteosarcoma is the most common heterologous element.

Immunohistochemistry, the carcinomatous component is universally positive for EMA and pan keratin and is positive for vimentin in $80 \%-90 \%$ tumours. Sarcomatous component reacts with vimentin only. In our case report, HPE demonstrated that the tumour was composed of spindle cells arranged haphazardly with focal hemangiopericytomatous and bone pattern suggestive of very aggressive and invasive behaviour.

\section{DISCUSSION OF MANAGEMENT}

Most patients with carcinosarcoma of urinary bladder had high histological grade and advanced stage at the time of presentation. These tumours have unfavourable prognosis, 
worse than high-grade urothelial carcinoma. Mean survival of patient is 1 - 2 years despite aggressive surgical management. Patients with regional and distant spread of disease have a 2fold and 8-fold increased risk of dying from sarcomatoid carcinoma of the bladder respectively. ${ }^{7}$ Pathological stage is the best predictor of survival in sarcomatoid variants. ${ }^{8}$ The only factor predictive of long-term survival are negative surgical margins and the absence of metastatic disease at the time of presentation. There is no standard treatment for sarcomatoid carcinoma due to rareness of tumour. Some investigators suggest neoadjuvant or adjuvant chemotherapy and/or radiotherapy; however, outcomes associated with CT/RT are inconsistent in large scale. Recently, gemcitabine with cisplatin show some positive effect on survival. In our case, partial cystectomy was done and followup was done up to 6 months, after which patient was lost for followup. Chemotherapy was advised to patient, but refused by the patient.

\section{FINAL DIAGNOSIS}

Sarcomatoid tumour of urinary bladder are rare and aggressive form of tumour with high recurrence rate. ${ }^{9} \mathrm{We}$ reported a case of bladder sarcoma treated with partial cystectomy. However, the treatment of choice is total cystectomy, but post-operative quality of life should be considered before taking any decision. Further studies and experience may provide more toward the treatment and increasing the survival.

\section{REFERENCES}

[1] Lopez-Beltran A, Pacelli A, Rothenberg HJ, et al. Carcinosarcoma and sarcomatoid carcinoma of the bladder: clinocopathological study of 41 cases. J Urol 1998;159(5):1497-503.

[2] Knappen BST, Uson AC, Melicon MM. Primary neoplasm occurring in vesicle diverticula: a case report of 18 cases. Urol 1960;83:153-89.

[3] Chen KT. 'Carcinosarcoma' of the bladder. Arch Pathol Lab Med 1992;116(8):811.

[4] Froehner M, Gaertner HJ, Manseck A, et al. Durable complete remission of metastatic sarcomatoid carcinoma of the bladder with cisplatin and gemcitabine in an 80-year-old-man. Urology 2001;58(5):799.

[5] Hosokawa Y, Kuwada M, Kumamoto H, et al. Case of sarcomatoid carcinoma of the urinary bladder. Hinyokika Kiyo 2008;54(8):561-3.

[6] Lopez-Beltran A, Cheng L. Histologic variants of urothelial carcinoma: differential diagnosis and clinical implications. Hum Pathol 2006;37(11):137188.

[7] Wright JL, Black PC, Brown GA, et al. Differences in survival among patients with sarcomatoid carcinoma, carcinosarcoma and urothelial carcinoma of the bladder. J Urol 2007;178(6):2302-7.

[8] Dahm P, Gschwend JE. Malignant non-urothelial neoplasms of the urinary bladder: a review. Eur Urol 2003;44(6):672-81. 